\title{
PROCESOS MORFOCLIMÁTICOS DOMINANTES EN LA PROVINCIA DE ALICANTE
}

\author{
Enrique Matarredona Coll
}

\section{RESUMEN}

El objeto del presente trabajo es poner de manifiesto la importancia del clima como agente de transformación del paisaje y su influencia sobre las formas y procesos geomórficos. A tal fin hemos aplicado a la provincia de Alicante el método de sistemas (Climate-Process Systems) propuesto por Wilson, en base a los datos termopluviométricos de 47 estaciones meteorológicas, que nos han permitido calibrar la distribución morfoclimática mensual y anual de cada estación, al tiempo que nos ha posibilitado la elaboración de un mapa de isodistribución morfoclimática provincial.

\begin{abstract}
The object of this study is to point out the importance of climate as an agent that transforms the landscape and the influence of climate in che geomorphic process. For this porpuse, we have applied to the Province of Alicante the Climate-Process System proposed by Wilson, based on temperature and rainfall data of 47 meteorologic distribution of each station monthly and in the year, it has also made possible the elaboration of a map of morphoclimatic isodistribution of the Province.
\end{abstract}

\section{Introducción}

La acción del clima sobre el relieve se manifiesta tanto en la explotación de la estructura geológica por la erosión diferencial como por los aspectos variables de su modelado. El análisis morfogenético es una técnica subjetiva por la cual se 
hacen correlaciones entre climas y formas de modelado. El clima, como factor morfogenético, incide sobre el paisaje imprimiéndole unas características que variarán en función de la litología, vegetación, acción antrópica, etc.

En efecto, el clima, como agente de transformación del paisaje, es determinante en cualquier ámbito geográfico, sin que ello signifique, en modo alguno, que ignoremos la influencia tectónica estructural que condiciona el tipo de material, discordancias litológicas... y toda una serie de elementos de singular importancia. La influencia del clima sobre las formas y procesos geomórficos han sido reconocidos desde hace mucho tiempo: el mismo Davis reconoció que los fenómenos climáticos modificaban el ciclo normal de erosión; siendo muchos, precisamente, los que con posterioridad han defendido la idea conceptual del clima como generador del modelado.

Aunque, evidentemente ello no signifique una total unanimidad, toda vez que ciertos investigadores en este campo, mantienen que las formas de modelado son esencialmente independientes del clima; postura que, obviamente cuestionamos puesto que parece imposible aislar una serie de parámetros (estructura, litología, historia tectónica, tiempo, etc.) de toda una serie de crisis climáticas interrelacionadas con las formas de modelado tanto heredadas como actuales.

Por otro lado, muchos son los criterios que pueden usarse para determinar los límites subjetivos y arbitrarios de un proceso morfoclimático. Así, la conjunción de los elementos termopluviométricos posibilita la caracterización de zonas climáticas que con diversos objetivos han sido postulados desde antaño por diversos autores (Köppen, Blair, Lang, Martonne, Emberger, Dantin-Revenga) y que han sido aplicados a nuestro entorno más próximo por diferentes investigadores (López Gómez, Albentosa, Clavero, etc.). Incluso, el empleo de otros criterios más complejos (Thornthwaite, Papadakis) no sólo permite la caracterización climática de una determinada región sino que permite cuantificar la importancia de la sequía o realizar una estimación sobre las necesidades hídricas del suelo, pero en ningún caso cuantifican y caracterizan las relaciones entre el clima y los procesos de modelado. Este es, precisamente, el objeto del presente trabajo, aplicar un índice que permita establecer esa correlación. A este respecto, pensamos que uno de los más conocidos, independiente de su sencillez de aplicación, es el método empleado por Wilson (1969): el método de sistemas (Climate-Process Systems), que Wilson propone, supone el análisis de los débitos pluviales junto al de las temperaturas, y constituye, pensamos, un criterio importante para valorar la potencialidad de esa relación clima-procesos en la provincia, a través del análisis de esos dos parámetros: precipitaciones y temperaturas.

El no tener en cuenta otras variables morfogenéticas (litología, estructura) -pese a lo afirmado- no resta valor a los resultados obtenidos, sino que creemos, que con su aplicación se puede alcanzar una aproximación-tipo morfoclimática 
bastante aceptable. El término sugerido por Wilson, Climate-Process System, matiza conexiones entre clima, procesos y formas de modelado, que pueden integrarse globalmente en el concepto sistema morfoclimático (Morfogenetic-Systems), y permite acuñar la idea de regiones morfogenéticas, siempre y cuando exista una relación actual, a nivel regional, entre esas variables: clima-procesos-formas de modelado.

Fundamentándose en la zonación climática del planeta, Wilson considera que dentro de los cuatro sistemas fundamentales -glaciar, árido, templado-húmedo, selva- se pueden concebir modelados bastante complejos resultantes de la interacción de alguno de ellos. Ese es el caso, por ejemplo, del sistema mediterráneo -directamente relacionado con el objeto de este trabajoen el que se distingue entre Árido, Semiárido y Templado-Húmedo.

Por otro lado, con independencia de la concepción estrictamente morfoclimática del método de Wilson, los umbrales de delimitación entre un sistema y otro son fijos, circunstancia bien alejada de la realidad donde el modelado se muestra más fluctuante, más vivo, no tan compartimentado. Con todo, el método nos parece apropiado por las ideas que aporta y puede ser un elemento de trabajo válido para realizar una aproximación a la distribución morfoclimática de la provincia de Alicante.

Procesos morfoclimáticos dominantes

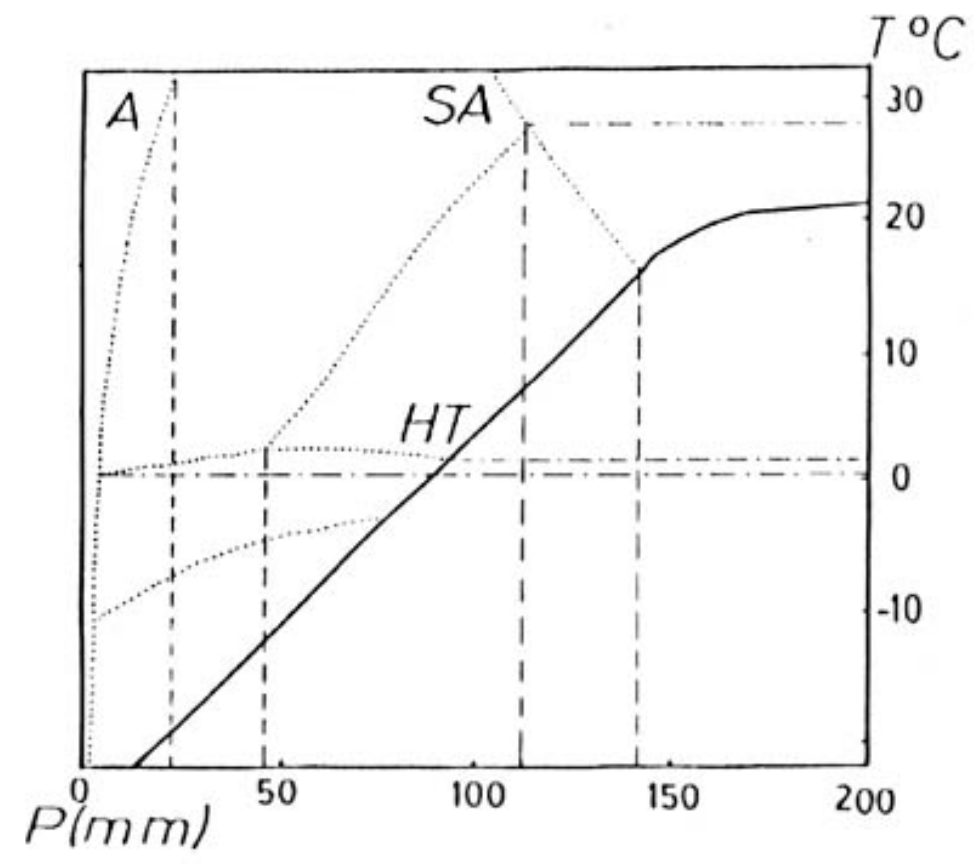

DIAGPAIA-TIPO, COH IHDICACIOH DE LOS UTBRALES TERHUPLUVIOHETRI COS UTILIZADOS EIH EL IIETODO DE WILSOH (CL IIAATE-PROCESS SYSTEH). A, ARIDU. Tenperaturas $\left(-23^{\circ} \mathrm{C}\right.$ a $\left.32^{\circ} \mathrm{C}\right)$. Precipitaciones $(1,3 \mathrm{a}$ 21 niu). SA. SEIIIMRIDO. Teniperaturas $\left(1,5\right.$ a $\left.32^{\circ} \mathrm{C}\right)$. Precipitacio nes $\left(4\right.$ a 116 ma.) HT. TEHPLADO-HUnEDO. Tenperaturas $\left(1,7\right.$ a $\left.27^{\circ} \mathrm{C}\right)$ Precipitaciones (46-142nai).

La aplicación de los criterios de Wilson a la provincia ha supuesto la recopilación de 
datos termopluviométricos de 47 estaciones que nos han permitido, una vez introducidos en el correspondiente diagrama de termohietas, calibrar la distribución morfoclimática mensual y anual de cada estación, así como la ulterior elaboración de un mapa de isodistribución morfoclimática provincial.

Con todo, en un ámbito tan restringido como el que nos ocupa, las variaciones climáticas latitudinales son de escasa importancia -pese a la sensible diferencia que se puede observar entre el sector septentrional y el meridional-. Así, según Rosselló (1965), se pueden distinguir tres zonas climáticas: una zona subhúmeda coincidente con las más altas tierras del prebético septentrional en las que su favorable situación a la influencia de la Baja balear posibilita unos umbrales pluviométricos comprendidos entre los 500 y $800 \mathrm{~mm}$., con unos niveles térmicos anuales situados entre $\operatorname{los} 12$ y $15^{\circ} \mathrm{C}$; un sector semiárido, que abarca la orla costera y ciertos valles interiores donde ya se alcanza, según la tipología de Thornthwaite, de 3 a 4 meses áridos, y en los que, pese a una dinámica similar, la localización geográfica matiza unos umbrales de humedad inferiores; y un espacio «extremadamente árido», según Lautensach-Mayer (1961), referido a las cuencas bajas del Vinalopó y Segura en las que la sequía estival es superior a los 5 meses y las precipitaciones no alcanzan la isoyeta de los $300 \mathrm{~mm}$.

En este punto, Juárez (1985) en aplicación de los criterios de Thornthwaite, afirma que «todos los municipios de la provincia demandan una necesidad de agua superior a los 700 $\mathrm{mm} / \mathrm{año}$, menos el espacio próximo a Sierra de Aitana que solo requiere $595 \mathrm{~mm} / \mathrm{año»,}$ circunstancia que viene a confirmar la diferencia entre los sectores señalados, con lo que podemos corroborar la variedad climática provincial desde las húmedas zonas de Aitana y parte del Marquesat hasta la aridez y secas tierras del Bajo Segura.

En función de los datos obtenidos, y siguiendo una distribución climática zonal, consideramos morfoclimáticamente a la provincia inserta en el sistema mediterráneo, con ciertos vestigios de continentalidad en su sector interior más septentrional, de forma que a unos veranos secos y cálidos, les sigue un otoño lluvioso y un invierno más seco que las estaciones equinocciales. Así, la evapotranspiración sobrepasa a la precipitación la mayor parte del año (JUÁREZ, 1985), y sólo en las alineaciones prebéticas septentrionales (Aitana, Mariola, Serrella, etc.), podemos encontrar un cierto superávit de agua en el suelo en los meses de mayor precipitación.

De todo ello deducimos la existencia en el ámbito alicantino de un sistema morfoclimático fundamental -semiárido- que se yuxtapone en determinados momentos del año y según sectores, bien al sistema árido, bien al templado-húmedo. Así, a la vista del mapa de isodistribución morfoclimática mensual, apreciamos un dominio del sistema semiárido, con una aridez más acentuada en el sector meridional-en torno a las lagunas de la Mata y de Torrevieja-, así 
como en ciertas áreas del Campo de Alicante y del Bajo Segura, en donde se observan de 3 a 5 meses con dominancia del sistema árido, mientras que en el sector septentrional la complejidad es mayor, con zonas en las que más de diez meses se caracterizan como semiáridas -Valle del Vinalopó, Campo de Alicante, Hoya de Castalla, entre otras- al lado de otras en las que a esa dominancia semiárida se yuxtaponen de dos a cuatro meses de tipología templado-húmeda -Valles de Alcoy, Marina Alta- o bien suponen un 50\% semiaridez/templado-húmedo, como es el caso del Vall de Laguart y zona de Pego y Denia.

A pesar de que no hayamos detectado, por los datos de observatorios meteorológicos utilizados, ningún sistema clima-procesos de tipo periglaciar -entre otras razones, por la ausencia de observaciones en áreas montañosas (excepto Aitana)- podemos observar manifestaciones periglaciares en las alineaciones prebéticas del $\mathrm{N}$ de la provincia en cotas comprendidas entre los 800 y $1.000 \mathrm{~m}$. (según los casos), en forma de canchales de ladera que presentan materiales heterométricos con clastos angulosos y poca matriz fina y que parecen predominantes en las estructuras calizo-dolomíticas, que según Mateu se muestran afectadas por una red de diaclasas, relacionadas con procesos comprensivos y distensivos, los cuales, junto con los fenómenos de fracturación, facilitan estas acciones periglaciares, que pueden verse favorecidas por fenómenos de disolución y acciones antrópicas (MORALES, 1983).

Y todo ello tendente a esas acumulaciones o depósitos de crioclastos que distinguimos en los piedemontes de ciertas estructuras montañosas provinciales (Aitana, Serrella, Bernia, etc.). A este respecto, Rosselló (1975) ha indicado la importancia que durante el Wurm más reciente pudieron tener la crioclastia y la termoclastia en estos ámbitos levantinos; precisamente la alternancia fase fría fase templada, de notables implicaciones morfogenéticas, y de fecha más reciente pudo acontecer en lo que algunos investigadores -Duplessy, Derruau- y el propio Rosselló han convenido en llamar «Little Ice Age».

En cualquier caso, estos procesos se manifiestan en una morfología variada: bien torrenteras que surgen por coalescencia de canturrales procedentes de fenómenos de descamación termoclástica coincidentes con aguaceros de cierta intensidad que determinan formas alargadas, a modo de lenguas, perpendiculares al aterrazamiento actual; en otros casos, como ocurre en Serrella -según Rosselló- los conos se presentan a modo de coladas de bloques evolucionados y relativamente bien alimentados; o bien surgen taludes extendidos a lo largo de acantilados a base de bloques angulosos de una variada gama de calibres, en los que se aprecia una mayor capacidad de desplazamiento en favor de los mayores, descansando el conjunto sobre un lecho de cantos pequeños, limos o margas.

Asimismo, con cierta frecuencia según Rosselló, se presentan los grèzes litées, fenómenos relictos favorecidos por la fragilidad de ciertas calizas, entendidos como formas integradas por pequeños elementos angulosos dispuestos en capas inclinadas alternantes con lechos más arcillosos, visibles en las laderas 
septentrionales de la Sierra de Aitana y en la carretera de acceso a la Font Rotja. Por otro lado, según Poser (1957), la extremada permeabilidad de las calizas, dominantes en la montaña alicantina, y la escasez de material fino, impide la proliferación de procesos solifluidales actuales (ROSSELLÓ, 1975), aunque sea significativa la colada solifluidal visible en las proximidades del Estrecho de Agres -fosilizada actualmente por un potente estrato arbóreo- o el deslizamiento rotacional de la zona de Benillup (LA ROCA, 1980) por las obvias implicaciones paisajísticas que conlleva.

Descendiendo en altura, pero sin abandonar las áreas montañosas citadas, aparece anualmente, durante varios meses, el sistema templado húmedo en el que el nivel de precipitaciones provee al suelo de una reserva hídrica durante todo el año; como indica Juárez, ciertas áreas del Marquesat de Denia y las elevaciones prebéticas de tipo medio «no sólo reciben más cantidad de precipitación anual sino que las temperaturas son más reducidas» -sobre todo en la montaña- lo cual determina una mayor efectividad de aquellas.

De este modo, la circulación del agua excedente contribuye a fenómenos de arroyada concentrada, vinculados a precipitaciones de tipo torrencial, que tienen un gran poder erosivo sobre todo al actuar sobre vertientes con altos ángulos de inclinación que posibilitan acciones de desprendimientos, transporte, e incluso, acumulación de los derrubios ocasionados. Todo ello puede contribuir, si las pendientes son acusadas, a la formación de taludes y cantiles, mientras que si aquéllas son más suaves, pueden generarse depósitos de vertientes (GUALDA, 1986).

Por otro lado, la litología calcárea de estas zonas de altitud media, junto con las disponibilidades hídricas ( + de $500 \mathrm{~mm} / \mathrm{año}$ ) favorecen los procesos de karstificación determinantes tanto de las formas exokársticas presentes en Mariola y Serrella, por ejemplo, como las clásicas formas endokársticas de la Sierra de Aitana. Incluso, en este punto, la influencia climática del pasado puede ser visible sobre los bloques calizos atacados por corrosión kárstica y según Dumas (1977) reflejo de un clima pasado más cálido y húmedo. Precisamente, la herencia de pulsaciones antiguas puede haber contribuido en esos procesos disolutivos y en el incremento de formaciones travertínicas por acumulación de carbonatos.

En la actualidad, el dominio morfoclimático en este sector permite calibrar por un lado el papel del agua en el proceso morfogenético tanto desde el punto de vista mecánico (fisuración, porosidad, corrosión), como químico (disolución de las calizas); con la particularidad de observar en los procesos de disolución kárstica una mayor capacidad de erosión en las aguas frías, con lo que en principio ya tenemos un elemento de conexión entre los parámetros termopluviométricos en lo que concierne a su función morfogenética.

Los procesos morfogenéticos conectados al sistema semiárido -dominante con mucho en la zona de estudio- son la acción de arroyada -de forma notable- y 
DISTRIBUCION MORFOCLIMATICA MENSUAL Y MMUAL DE LA PROVINCIA DE MLICANITE

\begin{tabular}{|c|c|c|c|c|c|c|c|c|c|c|c|c|c|}
\hline & $E$ & $\mathrm{~F}$ & $M$ & A & u & J & $\mathrm{JL}$ & A & $s$ & 0 & N & 0 & AHUAL \\
\hline Agost & SA & $S A$ & SA & $S A$ & SA & SA & A & A & SA & SA & SA & SA & $S A_{10} A_{2}$ \\
\hline Agres & $\mathrm{HT}$ & $S A$ & SA & HT & SA & SA & A & A & SA & HT & SA & HT & $\mathrm{HT}_{4} \mathrm{SA}_{6} \mathrm{~A}_{2}$ \\
\hline Aitana & SA & $S A$ & HT & HT & HT & SA & A & A & SA & HT & SA & $\mathrm{HT}$ & $\mathrm{HT}_{5} \mathrm{SA}_{5} \mathrm{~A}_{2}$ \\
\hline Alcolecha & HT & HT & SA & SA & SA & SA & A & A & SA & HT & HT & HT & $\mathrm{HT}_{5} \mathrm{SA}_{5} \mathrm{~A}_{2}$ \\
\hline Alcoy & $S A$ & SA & SA & $S A$ & SA & SA & A & A & $S A$ & HT & $S A$ & $\mathrm{HT}$ & $\mathrm{HT}_{2} \mathrm{SA}_{3} \mathrm{~A}_{2}$ \\
\hline Alicante & SA & SA & SA & SA & SA & SA & A & A & $S A$ & $S A$ & $S A$ & SA & $\mathrm{SA}_{10} \mathrm{~A}_{2}$ \\
\hline Almoradi & $S A$ & $S A$ & SA & SA & SA & SA & A & A & $S A$ & SA & SA & SA & $S A_{10} A_{2}$ \\
\hline Altea & SA & $S A$ & $S A$ & SA & $S A$ & $S A$ & A & A & SA & $S A$ & SA & SA & $S A_{10} \wedge_{2}$ \\
\hline Bañeres & SA & SA & SA & SA & $S A$ & SA & A & $S A$ & $S A$ & SA & SA & SA & $S A_{11} A_{1}$ \\
\hline Benefama & SA & SA & SA & SA & SA & $S A$ & A & SA & SA & HT & SA & HT & $\mathrm{HT}_{2} \mathrm{SA}_{9} \mathrm{~A}_{1}$ \\
\hline Bentarrés & SA & SA & SA & SA & SA & $S A$ & A & A & SA & HT & $S A$ & HT & $\mathrm{HT}_{2} \mathrm{SA}_{8} \mathrm{~A}_{2}$ \\
\hline Benfdorm & SA & SA & SA & SA & $S A$ & $S A$ & A & A & SA & SA & SA & SA & $S A_{10} A_{2}$ \\
\hline Benifallim & HT & SA & SA & SA & SA & $S A$ & A & $A$ & SA & HT & SA & HT & $\mathrm{HT}_{3} \mathrm{SA}_{7} \mathrm{~A}_{2}$ \\
\hline Benisa & $\mathrm{HT}$ & SA & SA & SA & SA & SA & A & A & SA & $\mathrm{HT}$ & SA & $\mathrm{HT}$ & $\mathrm{HT}_{3} \mathrm{SA}_{7} \mathrm{~A}_{2}$ \\
\hline Biar & SA & SA & SA & SA & $S A$ & $S A$ & A & SA & SA & SA & $S A$ & SA & $S A_{11} A_{1}$ \\
\hline Callosa de Ens. & SA & SA & SA & SA & SA & SA & A & A & $S A$ & HT & SA & HT & $\mathrm{HT}_{2} \mathrm{SA}_{8} \mathrm{~A}_{2}$ \\
\hline Castalla & SA & SA & SA & SA & SA & $S A$ & A & A & $S A$ & $S A$ & SA & SA & $S A_{10} A_{2}$ \\
\hline Catral & SA & SA & SA & SA & SA & SA & A & A & SA & $S A$ & SA & SA & $S A_{10} A_{2}$ \\
\hline Cocentaina & HT & SA & SA & $S A$ & SA & SA & A & A & SA & HT & SA & SA & $\mathrm{HT}_{2} \mathrm{SA}_{8} \mathrm{~A}_{2}$ \\
\hline Denia & HT & SA & HT & $S A$ & SA & SA & A & SA & SA & HT & HT & HT & $\mathrm{HT}_{5} \mathrm{SA}_{6} \mathrm{~A}_{1}$ \\
\hline Elche & $S A$ & $S A$ & SA & $S A$ & SA & SA & A & A & SA & SA & $S A$ & SA & $S A_{10} A_{2}$ \\
\hline Fontilles & HT & $S A$ & HT & HT & SA & SA & A & A & SA & HT & HT & HT & $\mathrm{HT}_{6} \mathrm{SA}_{4} \mathrm{~A}_{2}$ \\
\hline Gorga & HT & SA & SA & SA & SA & $S A$ & A & A & SA & HT & SA & HT & $\mathrm{HT}_{3} \mathrm{SA}_{7} \mathrm{~A}_{2}$ \\
\hline Guardamar & SA & $S A$ & SA & SA & SA & SA & A & A & $S A$ & SA & SA & SA & $S A_{10} A_{2}$ \\
\hline 161 & SA & SA & SA & SA & SA & SA & A & A & SA & SA & SA & SA & $S A_{10} A_{2}$ \\
\hline Jalon & HT & SA & SA & SA & SA & SA & A & $S A$ & $S A$ & $\mathrm{HT}$ & $\mathrm{HT}$ & HT & $\mathrm{HT}_{4} \mathrm{SA}_{7} \mathrm{~A}_{7}$ \\
\hline Jórea & SA & SA & SA & SA & SA & SA & A & A & 'SA & HT & SA & HT & $\mathrm{HI}_{2} \mathrm{SA}_{8} \mathrm{~A}_{2}$ \\
\hline Jijona & SA & $S A$ & SA & SA & SA & SA & A & A & SA & SA & SA & SA & $S A_{10} A_{2}$ \\
\hline Laguna La Mata & SA & $S A$ & A & SA & A & A & A & A & $S A$ & $S A$ & SA & SA & $\mathrm{SA}_{7} \mathrm{~A}_{5}$ \\
\hline Lag. Torrevieja & SA & SA & A & SA & A & A & A & A & $S A$ & $S A$ & SA & SA & $\mathrm{SA}_{7} \mathrm{~A}_{5}$ \\
\hline Monforte & SA & SA & SA & SA & SA & $\$ \wedge$ & A & $\wedge$ & $S A$ & SA & SA & SA & $\mathrm{SA}_{10} \mathrm{~A}_{2}$ \\
\hline Novelda & SA & $S A$ & $S A$ & $S A$ & SA & SA & A & A & SA & $S A$ & $S A$ & SA & $\mathrm{SA}_{10} \mathrm{~A}_{2}$ \\
\hline Orithuela & SA & SA & SA & SA & SA & SA & A & A & $S A$ & SA & SA & SA & $S A_{10} A_{2}$ \\
\hline Pego & SA & HT & "IT & HT & SA & SA & $A$ & SA & SA & HT & HT & $\mathrm{HT}$ & $\mathrm{HT}_{6} \mathrm{SA} \mathrm{A}_{5} \mathrm{~A}_{1}$ \\
\hline Pendguila & SA & SA & SA & SA & HT & SA & A & $S A$ & SA & HT & SA & HT & $\mathrm{HT}_{3} \mathrm{SA}_{8} \mathrm{~A}_{1}$ \\
\hline Pinoso & SA & SA & SA & sA & SA & SA & A & A & SA & SA & SA & SA & $S \Lambda_{10} A_{2}$ \\
\hline Relleu & SA & $S A$ & SA & SA & SA & SA & A & A & SA & $S A$ & SA & SA & ${ }^{S A}{ }_{10} A_{2}$ \\
\hline Rojales & SA & SA & $S A$ & SA & SA & SA & A & A & SA & SA & SA & SA & $S A_{10} A_{2}$ \\
\hline S.Miguel Salinas & SA & SA & SA & SA & SA & A & A & A & SA & SA & SA & SA & $\mathrm{SA}_{9} \mathrm{~A}_{3}$ \\
\hline Sella & SA & SA & $S A$ & SA & SA & SA & $\hat{A}$ & A & SA & HT & SA & SA & $\mathrm{HT}_{1} \mathrm{SA}_{9} \mathrm{~A}_{2}$ \\
\hline Tdrbena & HT & SA & HT & HT & SA & SA & A & $5 \wedge$ & SA & HT & SA & HT & $\mathrm{HT}_{5} \mathrm{SA}_{6} \mathrm{~A}_{1}$ \\
\hline Torremanz omas & SA & HT & SA & SA & HT & SA & A & SA & SA & $S A$ & HT & HT & $\mathrm{HT}_{4} \mathrm{SA}_{7} \mathrm{~A}_{1}$ \\
\hline Torrevieja & SA & SA & SA & SA & SA & A & A & A & SA & SA & SA & SA & $\mathrm{SA}_{9} \mathrm{~A}_{3}$ \\
\hline Vergel & HT & SA & SA & SA & SA & SA & A & SA & SA & HT & HT & HT & $\mathrm{HT}_{4} \mathrm{SA}_{7} \mathrm{~A}_{1}$ \\
\hline villajoyosa & SA & SA & SA & $S A$ & SA & SA & A & A & SA & SA & SA & SA & $S A_{10} A_{2}$ \\
\hline villena & $S A$ & SA & SA & SA & SA & $S A$ & A & A & $S A$ & $S A$ & $S A$ & SA & $S A_{10} A_{2}$ \\
\hline
\end{tabular}

HT: Templado-húmedo / SA: Semiarido / A: Arido 
la meteorización mecánica. El agua de arroyada incrementada en su acción erosiva por los recursos hídricos coetáneos con precipitaciones de tipo torrencial, junto a una litología adecuada a base de margas, arcillas y yesos, que se muestran bastante deleznables y con una escasa competencia, modelan un paisaje acarcavado con barrancos separados por agudas aristas (VAN ZUIDAM, 1976), que en ocasiones ofrecen un aspecto caótico, con un alto grado de abruptuosidad, mientras que en otras, las crestas se muestran más redondeadas, en función de la mayor o menor componente arcillosa de la estructura, así como de los niveles de pendiente y rexistasia que ofrezcan sus vertientes.

Así, sobre arcillas la escorrentía superficial va provocando incisiones verticales que determinan una serie de pináculos paralelos -Vinalopó, al N de Elche (BALLESTA, 1986)-. Sobre las margas, el escarpe muestra una cierta inclinación; la degradación de las mismas provoca acumulaciones y, según López Bermúdez (1973), los interfluvios que separan los canalillos presentan formas redondeadas, con un notorio retroceso de márgenes.

Mientras tanto, cuando los márgenes están formados por material aluvial -cantos y gravas con matriz arcillosa- las ramificaciones son profundas y los interfluvios aparecen como torrecillas convexas. Finalmente, sobre arcillas y yesos del Keuper triásico, surgen, según Nicod, procesos de karst salino-yesoso en los que se yuxtaponen la acción mecánica y la disolución (MATARREDONA, 1983).

Asimismo, bajo este sistema semiárido se observa la actuación de procesos de meteorización mecánica que, bien sea a través de fenómenos correlativos de humedad/secado -procesos de hidratación- o bien por fenómenos haloclásticos, posibilitan la desagregación lítica y la aparición de unas formas de modelado, a modo de alveolos o pequeñas concavidades, que aparecen en los escarpes, y que conocemos como taffonnis, los cuales, con el tiempo, pueden conducir a un retroceso paulatino de dichos escarpes -por desmoronamiento (según los materiales)- así como a una serie de depósitos limo-arenosos al pie de los mismos.

Bajo este mismo sistema morfogenético semiárido, es frecuente reconocer la existencia de unas superficies aplanadas, de suaves pendientes, que desde los retablos montañosos más o menos próximos tienden a converger en el fondo de los valles. Se trata de los glacis, término acuñado por Dresch en 1939 para referirse a estas superficies planas de escasa pendiente, situadas al pie de las montañas, después de múltiples controversias tendentes a distinguirlos del concepto pediment (Hernández Pacheco, Joli, McGee, Bryan, Tator, King, etc.) que llevaría en 1972 a Van Genderen a caracterizar a los glacis como formas típicas de medios semiáridos, mientras que, en cualquier caso, los pediments podrían considerarse como formas residuales en estos medios climáticos.

De todos modos, los glacis aparecen en la provincia como las formas de relieve más frecuentes; a veces escalonadas, fruto de una cierta poligenia, generalmente 

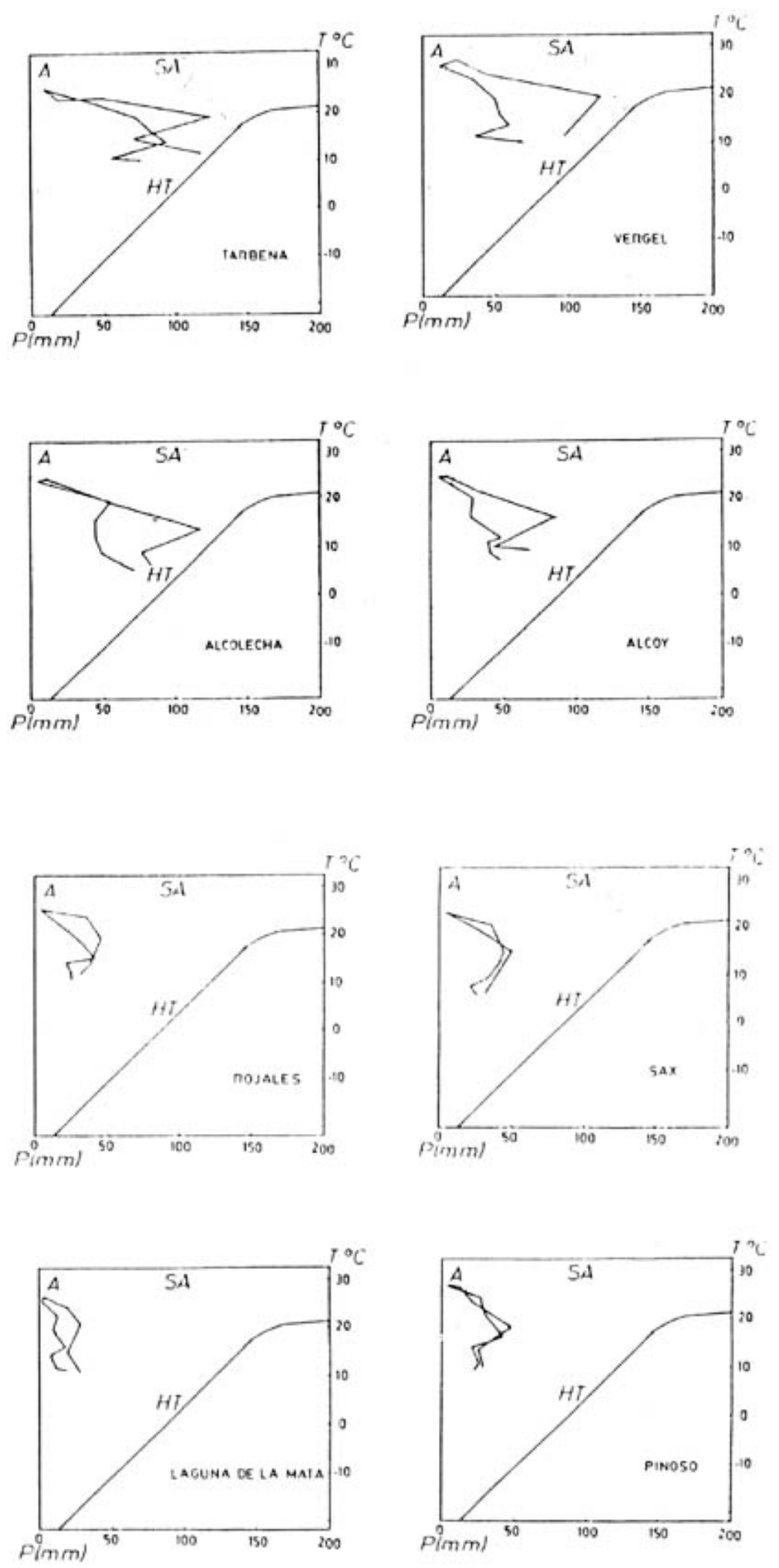

Sistenas Clima-Frocsos (Clinate-Frocess Systems) de ocho ectaciones neteorol6;icas de la provincia de Alicante. 
encostradas en caliche, incluso aislando entre ellas relieves de escaso vigor -como ocurre con los glacis del Campo de Alicante en relación a la Serra Grossa o Fontcalent, por citar dos casos significativos-; o dejando en resalte lambeaux, entendidos como retazos de niveles de glacis que quedan como testigo de superficies anteriores; aunque normalmente los glacis aparezcan diseccionados por ramblas y barrancos.

Abundan, como hemos indicado, los glacis de acumulación, en los que ya aparece un cierto principio de selección con gravas más maduras alternando con capas limoarenosas, e incluso, con encostramientos calcáreos. De todos modos, se trata de formaciones, tanto coluviales como abanicos torrenciales, que se superponen, yuxtaponen o se encajan entre sí, y aunque, generalmente, el espesor de estos glacis de acumulación no supera los $5 \mathrm{~m}$., en ocasiones pueden sobrepasar la decena (Muro de Alcoy, Benidorm, Laguna de Salinas).

Su elaboración genética parece que se corresponde, en su mayoría, con las últimas crisis climáticas y morfogenéticas pleistocenas; es decir, que los factores que pueden haber contribuido en su desarrollo pudieron ser los espasmos climáticos cuaternarios, que posibilitaron una fase rexistásica -asociada al descanso del volumen pluviométrico e intensa torrencialidad- y una fase fría que pudo posibilitar el incremento de la crioclastia, aparte del predominio calizo del sector, sin olvidar otros detalles como la coalescencia de conos de deyección o el endorreísmo interior de muchas áreas. En cualquier caso, las esporádicas escorrentías fluviales actuales pueden relacionarse con ciertos procesos de arroyamiento difuso sobre estas superficies, sin olvidar con ello las profundas disecciones a que están sometidos por ramblas y barrancos coincidentes con áreas de arroyamiento concentrado.

Por otro lado, en determinadas cuencas (Vinalopó y Busot), los depósitos fluviales y coluviales presentes en los glacis de acumulación muestran gran variedad heterométrica y altimétrica (MATARREDONA, 1983; BOX, 1983) consecuencia lógica de las irregularidades del régimen hidrológico, consecuente -como se ha indicado- con las fluctuaciones climáticas pleistocenas. Cuando se trata de zonas deprimidas -lagunas de Salinas y de Villena, valle de Benejama- amplias y poco accidentadas, suelen desarrollarse formas asociadas de glaciscono (Barranc de la Neu-glacis Solana de Benejama), formados por deposición de materiales procedentes de las vertientes y que en el caso de los conos de deyección han perdido poder de arrastre por modificaciones de pendiente de la red, con lo cual ofrecen estratificaciones con niveles alternantes de limos y cantos poco rodados, a veces encostrados en la superficie (MATARREDONA, 1983).

El sector meridional de la provincia, próximo al polo de aridez del SE peninsular, ofrece unas características climáticas con una clara indigencia pluviométrica y unas temperaturas elevadas que inciden en una profunda actividad de la ETP, al tiempo que el carácter espasmódico de las precipitaciones posibilita no sólo la alternancia estiajes-crecidas de la red hidrográfica, sino también el 
régimen funcional de las ramblas-barrancos con las naturales incidencias morfológicas, tanto en la evolución de los lechos como en los márgenes, al tiempo que la escasa densidad del tapiz vegetal y el predominio de materiales blandos facilita la labor de aguas de arroyada que dan lugar a valles desmesuradamente amplios en relación con los módulos absolutos y bajos coeficientes que normalmente les caracteriza.

Por otro lado, el aumento de la aridez que se observa lleva consigo la acentuación de los procesos que hemos visto presentes en zonas morfoclimáticamente semiáridas. Así, los glacis, que también aquí constituyen uno de los elementos más representativos, se encuentran profundamente entallados por ramblas y barrancos que denotan un intenso proceso de actividad erosiva, de tal modo que en ciertas áreas, la superficie encostrada del Cuaternario antiguo corta al Mioceno areniscoso y es disecada en cerros testigos y lanières (DUMAS, 1977).

Asimismo, unido a condicionamientos, en algún caso de tipo geológico, pero sobre todo de orden morfotectónico, se localizan fenómenos endorreicos, conectados en el caso de ciertas áreas -laguna de La Mata y Torrevieja- a procesos de restinga, en donde la mayor aridez se empareja con la marisma (ROSSELLÓ, 1969).

En efecto, las lagunas citadas, separadas del mar por un cordón arenoso litoral, aunque ofrecen un origen sedimentario marino, en su formación ha contribuido tanto el carácter depresivo del terreno como el cordón de la restinga que actúa de separación, el cual por su permeabilidad -debida a las areniscas pliocenas que lo configuran- ha favorecido los fenómenos de filtración hacia la depresión interior; y todo ello, además, en un área en la que los totales pluviométricos notablemente inferiores a los $300 \mathrm{~mm} /$ año posibilitan unos considerables valores de la ETP, hechos que permitirían perfectamente calificar a la zona como «subdesértica».

Además, el carácter salino de los niveles acuíferos filtrados, en un medio climático caracterizado por los altos valores de la ETP, explica los procesos de haloclastia que tienen su manifestación no sólo en la formación de solontchaks por las altas concentraciones salinas que se dan en los horizontes edáficos superficiales, sino también en el consiguiente aprovechamiento de tipo económico, a través de las instalaciones salineras de Torrevieja (COSTA, 1981).

Por otro lado, queremos significar que no hemos hecho referencia a la morfología litoral por su carácter azonal, a pesar de que pensamos, siguiendo la opinión de algunos investigadores en este campo (SANJAUME, 1985) que las peculiares características dinámico-climáticas de la cubeta mediterránea tienen su natural incidencia tanto en los movimientos marinos como en los consiguientes procesos de erosión y de sedimentación que configuran su perfil costero. 
REGIOIES MOROCLI WICAS DE IA RROVLCIA DE ALTCATE

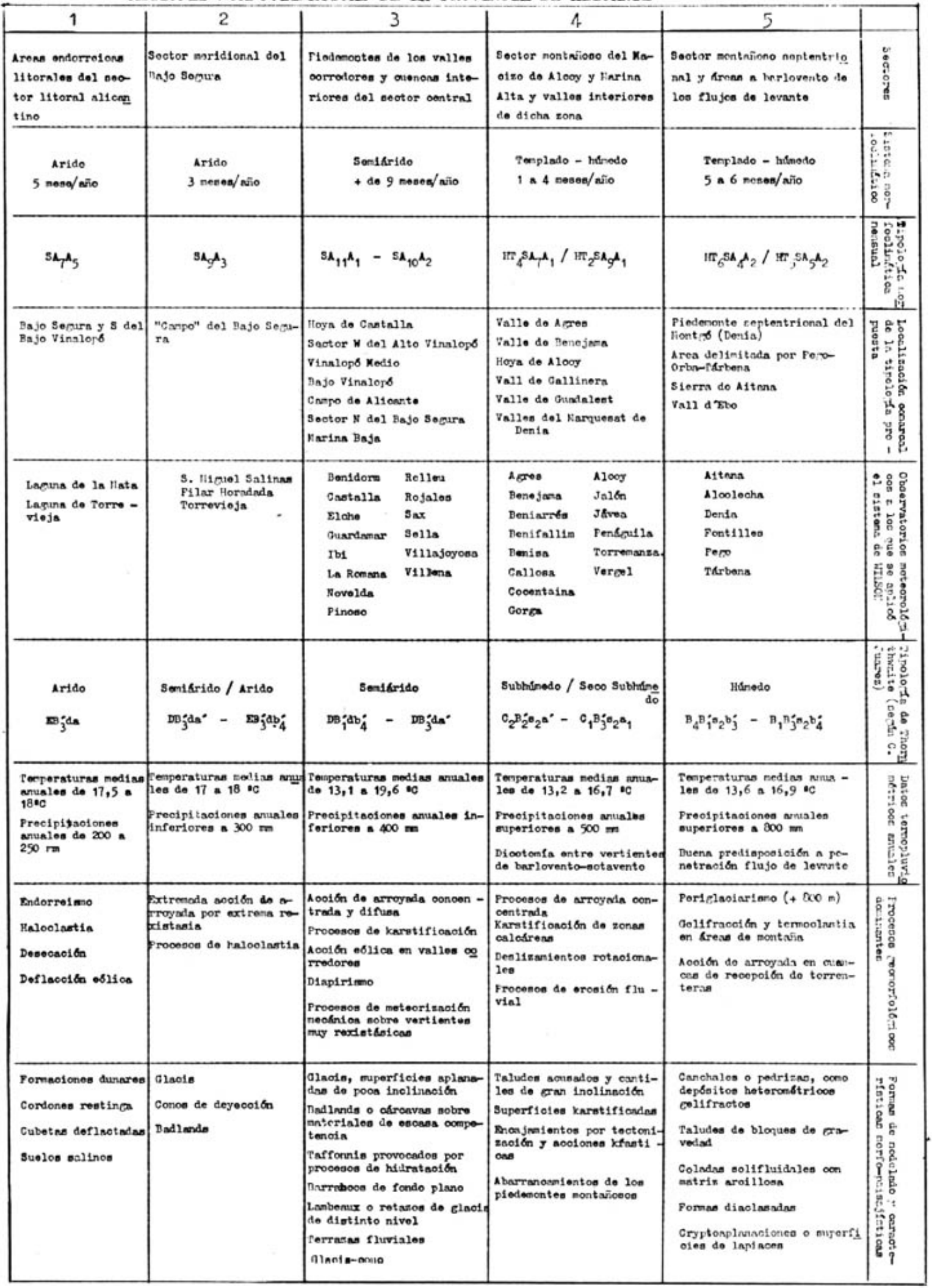




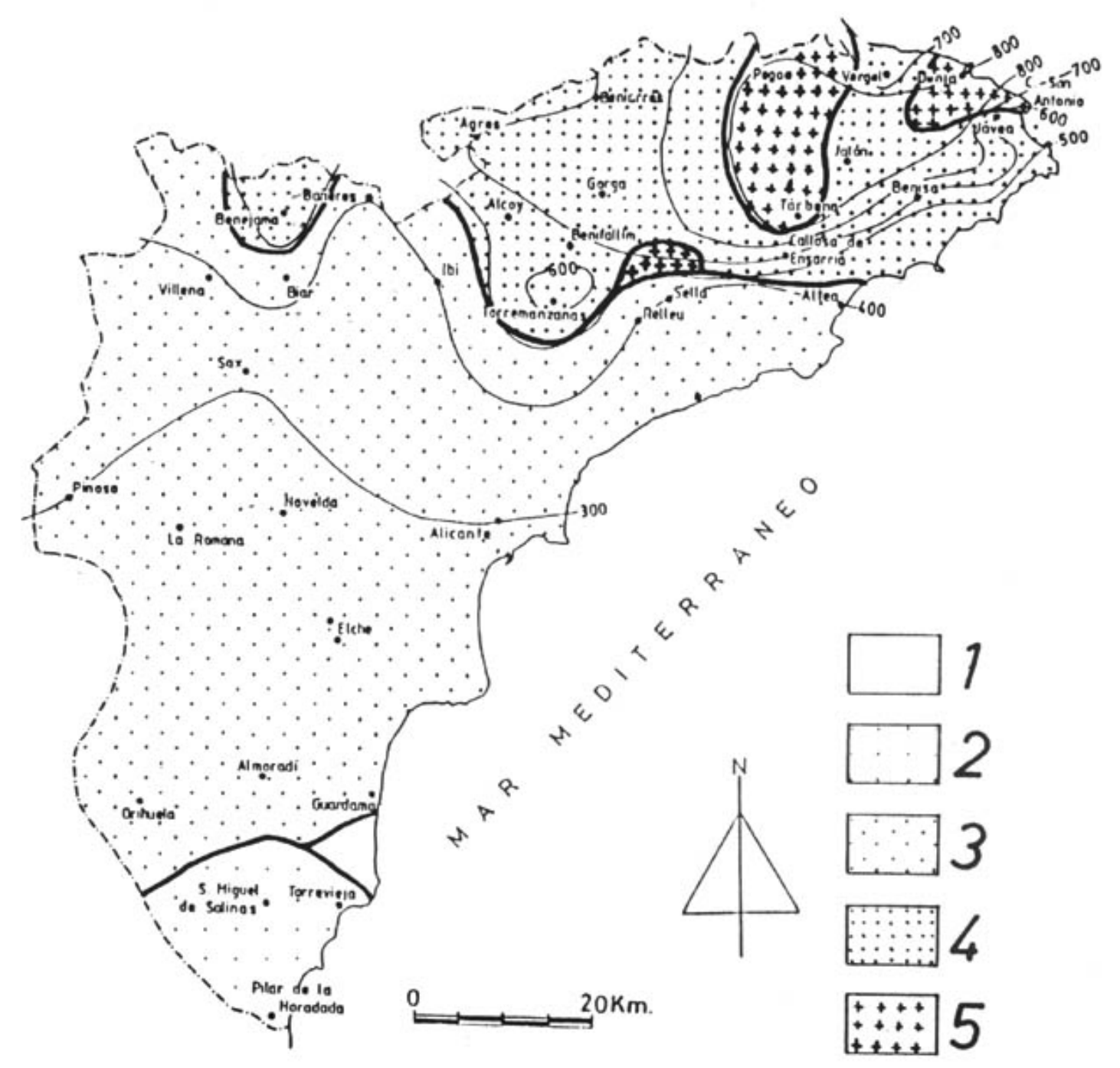

IMPA DE ISOUISIRIBUCIOH IOUROCL IMAIICA MEHSUAL (IIétodo de WILSOII)

1. Arido 5 meses al año. 2. Arido 3 meses al aío. 3. Semiárido más de 9 meses al año. 4. Templado-húmedo de 1 a 4 meses al año.

5. Templado-húmedo más de 5 meses al año. 


\section{Conclusiones}

La combinación entre precipitaciones y temperaturas no sólo permite establecer el mayor o menor grado de aridez provincial, sino que posibilita, a su vez, -en aplicación de la metodología de Wilson- concretar una tipología morfoclimática anual, que nos permite caracterizar en la provincia de Alicante las siguientes regiones o zonas morfoclimáticas:

1. Áreas endorreicas litorales del sector meridional alicantino. Corresponde a la tipología más árida $\left(\mathrm{SA}_{7} \mathrm{~A}_{5}\right)$ en la que cinco meses se caracterizan como áridos y que se localiza en una pequeña zona que cubren las lagunas de La Mata y Torrevieja $\left(\mathrm{EB}_{3} \mathrm{da}\right.$, según Thornthwaite) en la que las altas temperaturas y los escasos niveles pluviométricos explican, no sólo unos valores notables de la ETP, sino unos procesos geomorfológicos marcados por el endorreísmo, la haloclastia, la desecación, etc., que configuran un paisaje subdesértico, con notables formaciones dunares y profusión de suelos salinos, fruto de los procesos haloclásticos.

2. Sector meridional del Bajo Segura. Aún dentro de la tipología árida, calificada por Thornthwaite como $\mathrm{EB}_{3} \mathrm{db}_{4}$, este sector se diferencia del anterior, en aplicación del sistema de Wilson, por disponer sólo de tres meses caracterizados como áridos $\left(\mathrm{SA}_{9} \mathrm{~A}_{3}\right)$. Se localiza en lo que se suele conocer como «campo» del Bajo Segura (VERA, 1984), donde los procesos geomórficos dominantes siguen marcados por la aridez, con una notable acción de la arroyada, incrementada por las condiciones rexistáticas.

3. Piedemonte de los valles corredores y cuencas interiores del sector central. Se trata del área que supera con mucho el $60 \%$ de la superficie provincial, inserta dentro de un sistema morfoclimático -claramente semiárido más de 9 meses al año- dentro de una tipología que en aplicación de la metodología seguida hemos caracterizado como $\mathrm{SA}_{10} \mathrm{~A}_{2}$, y que, obviamente encaja con las consideraciones planteadas por Juárez (1985), al tipificar este sector como semiárido. Abarca el Valle del Vinalopó, Campo de Alicante, sector N del Bajo Segura, Hoya de Castalla, la Marina Baja, etc. Se trata de una zona en la que los procesos geomórficos dominantes son el agua de arroyada, tanto concentrada como difusa, y los procesos de meteorización mecánica que fortalecen su acción sobre unas vertientes muy rexistásicas. Todo ello posibilita que las formas morfo-paisajísticas más frecuentes sean los glacis, glacis-cono, badlands y barrancos de fondo plano.

4. Sector montañoso del Macizo de Alcoy y Marina Alta y valles interiores de dicha zona. Se trata de un área en la que se dan de dos a cuatro meses como templado-húmedos $\left(\mathrm{HT}_{4} \mathrm{SA}_{7} \mathrm{~A}_{1}-\mathrm{HT}_{2} \mathrm{SA}_{9} \mathrm{~A}_{1}\right)$, tipología presente, sobre todo, en los valles de Agres, Gallinera, Guadalest, Hoya de Alcoy y valle de Benejama; zona que termopluviométricamente ofrece unas temperaturas medias anuales entre $13 \mathrm{y}$ $16,5^{\circ} \mathrm{C}$ y unas precipitaciones anuales superiores a los $500 \mathrm{~mm}$, con una clara dicotomía entre los observatorios a barlovento o situados a barlovento o 
sotavento de los flujos húmedos. En cualquier caso, esos condicionamientos climáticos se conectan con unos procesos geomorfológicos en los que predomina la arroyada concentrada y la karstificación, que posibilitan unos paisajes -según la tipología del roquedo- con profusos abarrancamientos, taludes acusados, y a veces, extensas superficies karstificadas.

5. Sierra de Aitana y áreas a barlovento de los flujos de Levante. Es la zona más húmeda, tanto por condicionamientos orográficos (1.588 m de Aitana), como por la buena predisposición a la recepción de los flujos dominantes-húmedos de Levante (Vall d'Ebo, Pego, Tárbena, Orba, etc.), que no sólo explican unas precipitaciones superiores a los 800 $\mathrm{mm} / \mathrm{año}$, sino su propia tipología morfoclimática $\left(\mathrm{HT}_{6} \mathrm{SA}_{4} \mathrm{~A}_{2}\right)$ en la que el $50 \%$ del año los meses tienen un carácter templado-húmedo. Los procesos geomórficos dominantes, sobre todo en las inmediaciones de Aitana y áreas por encima de los 800/1.000 m se relacionan con el periglaciarismo, la gelifracción, la termoclastia, etc., que determinan unas formas de modelado -en estas áreas de montaña- a base de depósitos heterométricos sobre las vertientes o taludes de gravedad o cryptoaplanaciones (según la componente lítica), mientras que en las cotas más bajas los mejores condicionamientos templado-húmedos (menos rexistasia) hace disminuir los efectos de los procesos de arroyada.

\section{BIBLIOGRAFÍA}

BALlESTA, R., Morfología fluvial en el Valle del Vinalopó, Tesis de Licenciatura, inédita, 1986, 239 pp. mecan.

BIROT, P., «La geomorphologie quaternaire dans les regions mediterraneennes», Etudes françaises sur le Quaternaire, INQUA, VIII Congrès, 1969, pp. 173-192.

BOX, M., «Un aspecto del modelado de la Cuenca de Busot: los glacis», Investigaciones Geográficas, núm. 1, 1983, Universidad de Alicante, pp. 181-198.

BUDEL, J., Klima Geomorphologie, Gebrüder Borntraeger, Berlin-Stutgart, 1977, 304 pp.

COSTA, J., «El mayor complejo salinero de Europa: Torrevieja-El Pinós», Estudios Geográficos, Madrid, 1981, pp. 397-430.

COTTON, C. A, «Alternating Pleistocene morphogenetic systems», Geol. Mag., 95, 1958, pp. 125-136. CHOLLEY, A, «Morphologie structurale et morphologie climatique», Ann. Geogr., 49, 1950, pp. 321-335.

DERBYSHIRE, E., Climatic Geomorphology. Mac Millan, London, 1973, 296 pp.

DRESCH, J., Phénomènes karstiques II. Memoires et Documents. CNRS, Paris, 1975, 322 pp. 
DUMAS, B., Le Levant espagnol. La genèse du relief. Paris, 1977, 520 pp.

DUPLESSY, J. C., «L'Etude Quantitative des climats ancienes», Meteorologie, Paris, 1979, pp. 17-34.

GENDEREN, J. L. van, An investigation into the glacis concept. Departament of Geography. University of Shefield, 1972, $194 \mathrm{pp}$.

GUALDA, C., La Sierra de Mariola y los procesos de modelado. Tesis de Licenciatura, inédita, 1986, 383 fol. mec.

JUÁREZ, C., «Tipología climática de la provincia de Alicante», IX Reunión de Bioclimatología, Centro Experimental Zonas Áridas, CSIC, Almería, 1985 (en prensa).

LA ROCA, N., «Deslizamiento rotacional-colada de fango en los valles de Alcoi (Mas de Jordam Benillup)», Cuadernos de Geografía, $\mathrm{n}^{\circ}$ 26, Departamento de Geografía. Universidad de Valencia, Valencia, 1980, pp. 23-39.

LÓPEZ BERMúdEZ, F., La Vega Alta del Segura. Clima, Hidrología y Geomorfología. Departamento de Geografía, Universidad de Murcia, 1973, 288 pp.

MATARREDONA, E., El Alto Vinalopó. Estudio Geográfico, Instituto de Estudios Alicantinos, Diputación Provincial, 1983, 370 pp.

MATARREDONA, E., «Circulación de las aguas y dificultades de avenamiento en la cuenca alta del Vinalopó», Estudios Geográficos, 175, CSIC, 1984, pp. 193-213.

MORALES, A. y otros, «Morfología de la Sierra de Bernia», Investigaciones Geográficas, $\mathrm{n}^{\circ}$ 1, Universidad de Alicante, 1983, pp. 117-147.

NICOD, J., Pays et paysages du calcaire. PUF, Paris, 1972, 244 pp.

PELTIER, L., «The geographic cycle in periglacial regions as it is related to climatic geomorphology», Ann. Assoc. Amer. Geogr., 40, 1950, pp. 214-236.

POSER, H., «Klimamorphologische Probleme auf Kreta», Zeitschrift für Geomorphologie, NF, 1 (1957), pp. 113-142.

ROSSELLÓ, V. M., «Distribución de cultivos en la provincia de Alicante», Cuadernos de Geografía, n 2, Valencia, 1965, pp. 129-166.

ROSSELLÓ, V. M., El litoral valencià, Valencia, L’Estel, 2 vols., 1969.

ROSSELLÓ, V. M., «Los canchales de montaña calcárea y los fenómenos termoclásticos», Actas de la II Reunión Nacional del Grupo Español de Trabajo del Cuaternario, Jaca, 15-20 de sept., 1975, pp. 223-235.

SANJAUME, E., Las costas valencianas. Sedimentología y morfología, Universidad de Valencia, Sección de Geografía, 1985, 505 pp.

TRICART, J. y CAILLEUX, A., «Morphogenic Systems and Morphoclimatic Regions», Climatic Geomorphology, (E. Derbyshire, edit.), Mac Millan, London, 1973, pp. 228-268.

TRICART, J., Precis de Geomorphologie. Geomorphologie Climatique, t. III, SEDES, Paris, 1981, 313 pp.

VERA, F., Tradición y cambio en el campo del Bajo Segura, Instituto de Estudios Alicantinos, Alicante, 1984, 219 pp.

WILSON, L., «Morphogenic classification», Encyclopedia of Geomorphology, (R. W. Fairbridge), Reinhold, New York, 1968, pp. 717-731.

ZUIDAM, R. A. van, Geomorphological development of the Zaragoza region. Spain, ITC, Enschede, 1976, $211 \mathrm{pp}$. 